\section{Leukozytenphosphatase, alkalische}

H. Baum

Institut für Laboratoriumsmedizin, Mikrobiologie und Blutdepot, Regionale Kliniken Holding RKH GmbH, Ludwigsburg, Deutschland

Synonym(e) Alkalische Neutrophilenphosphatase

Englischer Begriff leukocyte alkaline phosphatase

Definition Enzym der neutrophilen Granulozyten, das die Hydrolyse von Phosphatestern im alkalischen Milieu katalysiert.

Beschreibung Der Nachweis der alkalischen Leukozytenphosphatase in den neutrophilen Granulozyten dient der Abgrenzung einer chronisch myeloischen Leukämie (CML) von anderen Erkrankungen des myeloproliferativen Formenkreises oder entzündlichen und anderen tumorösen Prozessen. Der Stärkegrad der Färbung wird in 5 Intensitätsklassen (0-4) eingeteilt:

- $0=$ keine Reaktion
- 1 = homogene blasse Farbschleier

- 2 = zunehmend granuläre Farbniederschläge

- 3 = kräftig schwarzbraune Präzipitate

- 4 = maximale Schwärzung des Zytoplasmas

Es werden 100 segmentkernige neutrophile Granulozyten beurteilt, den Intensitätsstufen zugeordnet und mit dem Klassenfaktor multipliziert. Die Addition ergibt dann den Färbeindex (ALLP-Index, dimensionslos). Der Referenzbereich ist 10-100.

Werte $<10$ sind für die aktive Krankheitsphase einer CML typisch. Selten können hämolytische Anämien, eine Eisenmangelanämie oder eine Virusinfektion ähnlich tiefe Werte zeigen.

Werte $>100$ können bei entzündlichen Prozessen und in $>70 \%$ der Fälle einer Polycythaemia vera gefunden werden.

\section{Literatur}

Nerl C (1993) Zellen der Granulozytopoese - Funktionen und Lebensäußerungen der Granulozyten. In: Begemann H, Rastetter J (Hrsg) Klinische Hämatologie, 4. Aufl. Georg Thieme Verlag, Stuttgart, S 70-74 